\title{
Widely Linear Filtering for Multi-Impairment Compensation in Coherent Optical Systems
}

\author{
Rekha Yadav, Lakshmi Narayanan Venkatasubramani, R David Koilpillai, Member, IEEE, \\ Deepa Venkitesh*, Senior Member, OSA
}

\begin{abstract}
We propose a blind joint equalization algorithm for M-QAM signals based on a widely linear filtering approach. The proposed scheme jointly compensates receiver IQ imbalance and polarization mixing, along with carrier recovery, followed by transmitter IQ imbalance compensation. We first investigate the proposed scheme's tolerance to transceiver IQ Imbalance, polarization mixing, phase noise and frequency offset through numerical simulations for 32 GBd PM-16QAM and PM-64QAM signals and compare its performance with the conventional digital processing algorithms. Further, with the proposed algorithm, we experimentally demonstrate the improvement in $Q^{2}$ value to up to $\approx 1.22 \mathrm{~dB}$ for a $32 \mathrm{GBd}$ PM-16QAM and $\approx 3.72 \mathrm{~dB}$ for a 16 GBd PM-64QAM signal with a phase imbalance of $9^{\circ}$. We show that the MSE convergence of the proposed joint equalizer is much faster than conventional DSP algorithms. Deployment of such an equalizer in optical communication systems is beneficial due to its improved tolerance to multiple impairments, albeit with increased complexity.
\end{abstract}

Index Terms-Augmented signal processing, IQ imbalance, Joint Equalization, Modified widely linear CMA filter, Polarization demultiplexing, Widely linear processing

\section{INTRODUCTION}

The capacity of fiber optic communication systems is being scaled with higher-order modulation formats, multi-carrier modulations and with the use of wavelength and space division multiplexing schemes. In the case of single-carrier systems, the data rates can be increased by employing higher-order modulation formats coupled with advanced equalization algorithms implemented through digital signal processing (DSP) [1]. Despite the progress made in recent years on increasing the end-to-end signal-to-noise ratio (SNR) of transmission systems so that they are compatible with higher-order QAM formats, the generation and detection of ultra-high order QAM signals remain challenging [2]. Non-idealities in the operation of the optical IQ modulator such as IQ phase imbalance, IQ gain imbalance, and IQ skew pose stringent limitations for higher cardinality modulations and thus ultimately limits the capacity scaling [3]. Thus, these systems require either excellent and stable bias controls and calibration or need compensation at the receiver. In addition, the coherent receiver also poses similar challenges due to IQ gain and phase imbalance and IQ skew. There have been several studies done regarding the in-factory calibration of transmitter, receiver, or transceiver IQ imbalance and skew or

Rekha Yadav, Lakshmi Narayanan Venkatasubramani, R David Koilpillai, Deepa Venkitesh are with the Department of Electrical Engineering, Indian Institute of Technology Madras, Chennai, 600036, India.

Corresponding author*: Deepa Venkitesh (deepa@ee.iitm.ac.in). estimating them offline for calibration purposes [4]-[7]. These non-idealities are additionally influenced by temperature and other environmental conditions and hence requires dynamic calibrations for minimizing the consequent errors [9]. Several studies were done on the compensation of transmitter or receiver IQ imbalances separately, including Gram-Schmidt ortho-normalization process (GSOP), methods employing second-order moment estimation, use of real-valued MIMO adaptive filters, blind source separation techniques, and the use of Kalman filters [10]-[18]. The presence of IQ imbalance breaks the orthogonality between in-phase and quadrature components of the signal, and the signal becomes non-circular. The non-circular nature of the signal allows us to use augmented complex signal processing that is linear in signal and conjugate of the signal. IQ imperfections originating both at the transmitter and the receiver can also be modelled as a linear sum of signal and its conjugate and can be corrected using a complex widely linear filter (WLF) [19]. This approach is explored for receiver imbalance compensation in [20], and authors have also explored their work for transmitter imbalance in [21], without considering the effect of transmitter and receiver phase noise.

When there are multiple impairments to be compensated in a system, it is prudent to consider joint equalization for efficient realization [22]. We have recently demonstrated one such joint equalization method to track the phase noise, polarization, and IQ imbalance compensation using complex ModifiedWLF [23]. In the previous demonstrations of IQ imbalance compensation reported, the origin of impairment was considered either at the transmitter or the receiver. However, both of these subsystems simultaneously contribute to the impairment in a practical system. Recently, few studies for the estimation and compensation of transceiver imbalance in the single-polarization system were reported [24], [25]. In the ref. [24] machine learning techniques are used for separation, estimation, and compensation of transmitter and receiver imbalance. In contrast, authors in [25] have compensated receiver imbalance using standard GSOP and transmitter imbalance with a geometric construction method, which is a machine learning technique, to reduce the complexity. However, GSOP requires data buffering, leading to latency in processing and challenges for parallel implementation [8]. Authors in [26] have modelled and derived the interaction between transceiver imbalance and fiber link and found the transceiver imbalance values for in-service calibration. However, the complexity of the discussed model is very high, and the proposed scheme was 
not investigated experimentally. Recently, authors in [27] have compensated multi-impairments of transmitter and receiver for dual-polarization systems using multi-layer linear and widely linear deep learning techniques. However, the method discussed by them depends on pilots, and the scheme's complexity is much higher. In this work, we propose a blind, adaptive and joint compensation of polarization mixing, transceiver gain and phase imbalance, and carrier recovery based on minimum mean square error criterion of real and imaginary components of the signal independently, and this can be used in any dispersion-managed coherent optical communication system. We have investigated the proposed joint compensation of multi-impairments numerically and experimentally for PM16QAM and PM-64QAM modulated signals.

The mathematical model of IQ imbalance at transmitter and receiver and their effect on the received symbols is first discussed in Section II. In Section III, we discuss the widely linear filter and its limitations in compensation of IQ imbalanced signals in the presence of phase noise. We further propose a joint adaptive equalizer for multi-impairment compensation in the dispersion-managed optical communication system in Section IV. In Section V, we present the results of numerical simulations for 32 GBd PM-16QAM and PM-64QAM in the presence of all impairments and compare the performance of proposed transceiver DSP with the conventional DSP algorithms. In Section VI, we present the results of experiments validating our proposed algorithm for $32 \mathrm{GBd}$ PM-16QAM and 16 GBd PM-64QAM signals, and demonstrate the efficacy of our proposed algorithm in bandwidth-limited practical systems. In Section VII, we evaluate the computational complexity of the proposed algorithm and compare it with the conventional DSP algorithm, and in Section VIII, we conclude our findings.

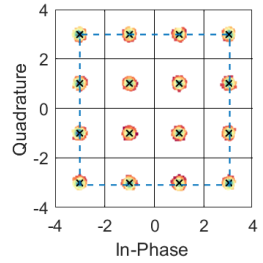

(a) No Imbalance

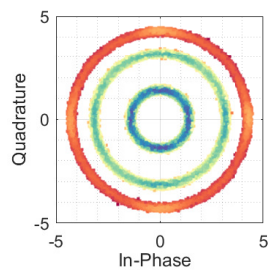

(g) No imbalance

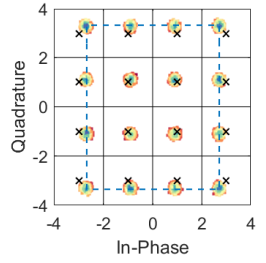

(b) $\epsilon_{t x}=0.1$

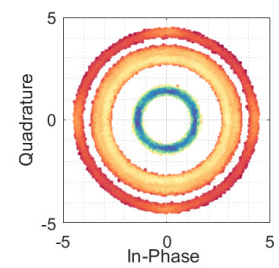

(h) $\epsilon_{t x}=0.1$

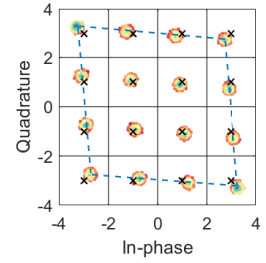

(c) $\phi_{t x}=10^{\circ}$

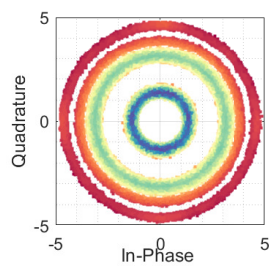

(i) $\phi_{t x}=10^{\circ}$

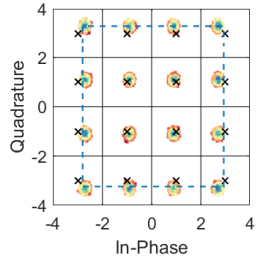

(d) $\epsilon_{r x}=0.1$

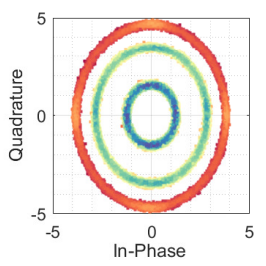

(j) $\epsilon_{r x}=0.1$

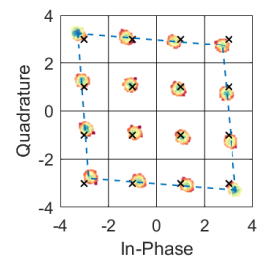

(e) $\phi_{r x}=10^{\circ}$

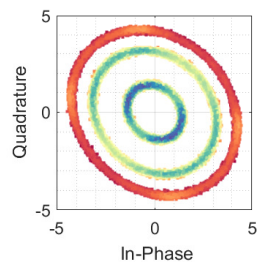

(k) $\phi_{r x}=10^{\circ}$

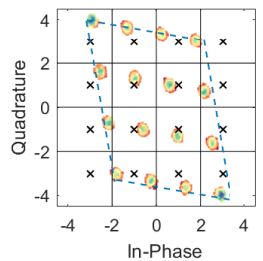

(f) $\epsilon_{t r}=0.1, \phi_{t r}=10^{\circ}$

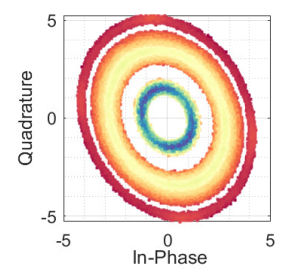

(1) $\epsilon_{t r}=0.1, \phi_{t r}=10^{\circ}$

Fig. 1: Effect of the transmitter and receiver IQ imbalance on 32 GBd 16QAM signal constellations. Upper row (a-f): Without any additional phase impairments, Lower row (g-1): With frequency offset of $100 \mathrm{MHz}$ and laser linewidth of $25 \mathrm{kHz}$ as additional phase impairments, $\epsilon$ : Gain imbalance factor, $\phi$ - Phase imbalance factor, $t x$-transmitter, $r x$ - receiver and $t r$ - both transmitter and receiver. 
to RF amplifiers that drive the IQ modulators. These amplifiers could pose different gains for the in-phase and quadraturephase signals, resulting in different input voltage swings at the modulator I and Q arms. Improper adjustments or drifting bias voltages of the IQ modulator and/or any RF path mismatch causes IQ phase imbalance in the transmitted signal. Similarly, receiver imbalance is caused either due to gain mismatch in the balanced photo-detectors or trans-impedance amplifiers, RF path mismatch, or imperfection in $90^{\circ}$ hybrid of the coherent receiver. Figure 1 shows the simulated 16QAM constellations ( 32 Gbaud, OSNR $=35 \mathrm{~dB}$ ) to portray the effect of transmitter and receiver imbalances; the top row shows the effect of imbalances without any other phase impairments, while the bottom row shows these along with transceiver phase noise (total linewidth $=25 \mathrm{kHz}$ ) and frequency offset of $100 \mathrm{MHz}$ between the transmitter and local oscillator laser. We observe that, in the absence of any additional phase impairments, the effect of transmitter and receiver imbalances on the constellation look identical in the upper row of Fig. 1. However, in the presence of frequency offset and phase noise, IQ imbalance impacts the constellations differently, as shown in the lower row of Fig. 1. Similar distortions due to gain imbalance shown in Fig. 1(b) and 1(d) and that due to phase imbalance shown in Fig. 1(c) and 1(e) at transmitter and receiver indicate that, these distortions are commutative. However, this commutative property is not maintained in the presence of laser phase noise and frequency offset, as indicated in Fig. 1(h) and 1(j) for gain imbalance and Fig. 1(i) and 1(k) for phase imbalance. Gain and phase imbalance at the transmitter results in broadening circles and additional concentric circles in the constellation, while receiver phase imbalance introduces rhombic transformations leading to an ellipticity in the constellation. The presence of IQ imbalances, both at the transmitter and the receiver, along with other transceiver phase impairments makes equalization more complex and challenging.

\section{A. Receiver IQ Imbalance Model}

We now consider the mathematical model to represent the effect of receiver IQ imbalance on the transmitted signal. Let $E_{r x}(t)$ represent the electric field of the received modulated signal affected by the transmitter and channel impairments $E_{L O}(t)$ represent the electric field of the local oscillator (LO) laser with unit average power, and $F_{f o}$ be the frequency detuning of the local oscillator laser with respect to the signal carrier frequency. Then, the I and Q components of the output of the coherent receiver $\left(I_{o}(t)=I_{i}(t)+j I_{q}(t)\right)$ can be represented respectively as,

$$
\begin{aligned}
I_{i}(t) & =\operatorname{Re}\left\{E_{r x}(t) E_{L o}^{*}(t)\right\} \\
\text { and, } I_{q}(t) & =\operatorname{Im}\left\{E_{r x}(t) E_{L o}^{*}(t)\right\} \\
\text { where, } E_{r x}(t) E_{L o}^{*}(t) & =E_{r x}(t) e^{-j \theta_{r x}(t)} e^{-j 2 \pi F_{f o} t}
\end{aligned}
$$

where, $\theta_{r x}(t)$ represents the phase noise of the local oscillator laser. The phase noise of the transmitter is included in the complex signal $E_{r x}(t)$. Let, $I_{r x}(t)$ be the output of
IQ imbalanced coherent receiver which can be modeled in baseband as

$$
I_{r x}(t)=\mu_{r x} I_{o}(t)+\nu_{r x} I_{o}^{*}(t) .
$$

Substituting $I_{o}(t)$ in terms of $E_{r x}(t)$,

$$
\begin{aligned}
I_{r x}(t) & =K_{1} E_{r x}(t)+K_{2} E_{r x}^{*}(t) \\
\text { where, } K_{1} & =\mu_{r x} e^{-j\left(2 \pi F_{f o} t+\theta_{r x}(t)\right)} \\
K_{2} & =\nu_{r x} e^{j\left(2 \pi F_{f o} t+\theta_{r x}(t)\right)}
\end{aligned}
$$

and, $\mu_{r x}$ and $\nu_{r x}$ are coefficients defined similar to those given in Eqn. (3) and (4) where $\epsilon$ and $\phi$ are defined at the receiver. For the impairments in $I_{r x}(t)$ to be compensated, $K_{1}$ should be made independent of time and $K_{2}$ should be made as close to zero to achieve complete equalization. Since $K_{2}$ is also time varying (from Eqn. 11), absolute suppression of $K_{2}$ is challenging.

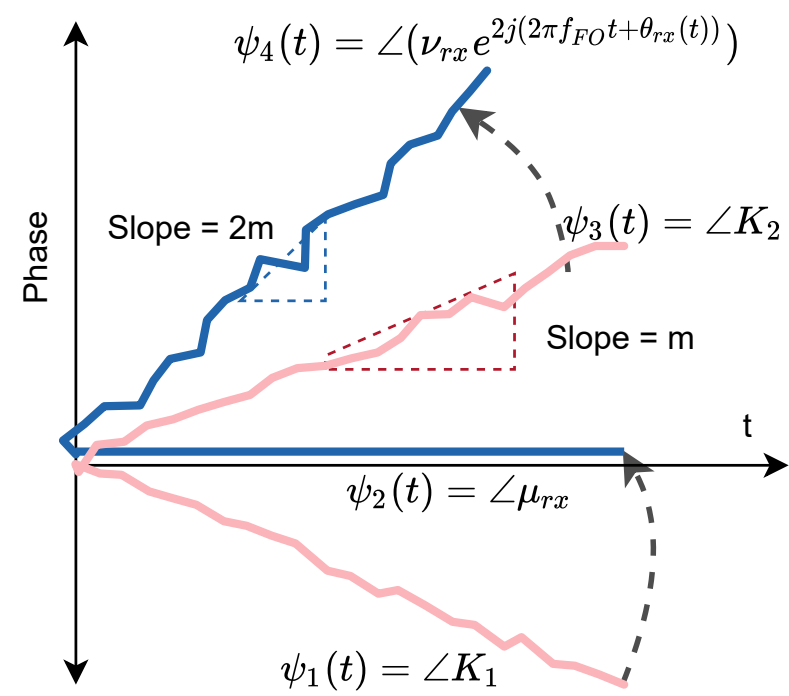

Fig. 2: Illustrative representation of evolution of unwrapped phase of the receiver IQ imbalanced signal as a function of time. $\psi_{1}$ and $\psi_{3}$ represent the time evolution of phase in the coefficients of $E_{r x}$ and $E_{r x}^{*}$ in the Eqn. 9. $\psi_{2}$ and $\psi_{4}$ represent the time evolution of phase in the coefficients of $E_{r x}$ and $E_{r x}^{*}$ in the augmented representation of the $I_{r x}(t) e^{j\left(2 \pi F_{f o} t+\theta_{r x}(t)\right)}$ signal.

Figure 2 shows the representative phase evolution of the different coefficients described in Eqn. (9). The terms $\psi_{1}$ and $\psi_{3}$ represent the phase evolution over time of the coefficients $K_{1}$ and $K_{2}$ respectively. This phase evolution slope $(\mathrm{m})$ indicates the instantaneous frequency offset along with the phase noise. Consider frequency offset and phase noise compensation as the first operation (without compensation of IQ imbalance) by multiplying the signal, $I_{r x}(t)$ with $\exp \left(j\left(2 \pi F_{f o} t+\theta_{r x}(t)\right)\right)$. Let, $\psi_{2}$ and $\psi_{4}$ represent the phase evolution of the coefficients of IQ imbalanced signal, $I_{r x}(t) e^{j\left(2 \pi F_{f o} t+\theta_{r x}(t)\right)}$. We can observe that $\psi_{2}(t)$ is independent of time and corresponds to the phase introduced due to imbalance. In contrast, phase error in $\psi_{4}(t)$ accumulates further, with its slope getting double. Therefore, carrier frequency and phase are not fully recovered, 
and conjugate signal interference becomes more significant due to an increase in the slope of $\psi_{4}(t)$. Hence, receiver IQ imbalance needs to be compensated before carrying out the carrier frequency and phase recovery. This same understanding is valid for the compensation of receiver IQ imbalance in the presence of other transceiver and channel impairments. Thus, receiver IQ imbalance compensation is required to be carried out prior to compensation of any other transceiver and channel impairments. It is also evident from equations 10 and 11 that $K_{1}$ and $K_{2}$ are very fast varying time-dependent components, leading to the additional complexity in equalization.

\section{B. Transmitter IQ Imbalance Model}

We now consider the effect of IQ imbalance on the signal originating at the transmitter. The output field $E_{i q}(t)$ of a balanced IQ Modulator in base-band can be written as $E_{i q}(t)=E_{o}(t) e^{-j \theta_{t x}(t)}$ where, $E_{o}(t)$ is the modulated signal and $\theta_{t x}(t)$ is the time varying phase noise introduced by the transmitter laser source. We can model the effect of transmitter IQ imbalance on the signal as

$$
E_{t x}(t)=\mu_{t x} E_{i q}(t)+\nu_{t x} E_{i q}^{*}(t)
$$

where, $\mu_{t x}$ and $\nu_{t x}$ are similar to those given in Eqn. (3) and (4), but defined at the transmitter.

Expanding $E_{i q}(t)$,

$$
E_{t x}(t)=\mu_{t x} e^{-j \theta_{t x}(t)} E_{o}(t)+\nu_{t x} e^{j \theta_{t x}(t)} E_{o}^{*}(t) .
$$

Rewriting the above equation,

$$
\begin{aligned}
E_{t x}(t) & \left.=K_{3} E_{o}(t)+K_{4} E_{o}^{*}(t)\right) \\
\text { where, } K_{3} & =\mu_{t x} e^{-j \theta_{t x}(t)}, \text { and } \\
K_{4} & =\nu_{t x} e^{j \theta_{t x}(t)},
\end{aligned}
$$

the IQ imbalance construct looks similar to that of the receiver imbalance. In the presence of transmitter laser phase noise, $K_{3}$ and $K_{4}$ are time-dependent IQ imbalance parameters. The IQ imbalanced transmitted signal $E_{t x}(t)$ further gets affected by channel and receiver impairments. Therefore, it is imperative to correct all the other impairments before equalizing transmitter IQ imbalance. It can also be gathered that the transmitter laser phase needs to be recovered only after correcting for transmitter IQ imbalance. However, it is impossible to distinguish the phase noise effect from signal laser and LO. Hence, a joint equalization for the transmitter IQ imbalance and phase recovery needs to be performed [23].

\section{Transceiver IQ Imbalance Model}

In this subsection, we consider the effect of IQ imbalances originating from both the transmitter and the receiver. In this model, we also consider the channel state information denoted by $H(t)$. The modelling of IQ imbalance is independent of polarization, and the channel matrix $H(t)$ includes the effect of polarization mixing and polarisation mode dispersion. We now model the transceiver IQ imbalance from the mathematical results drawn from the previous subsections, considering the same notations as discussed in previous subsections. The received field $E_{r x}(t)$ can be represented in terms of $E_{o}$ as

$$
E_{r x}(t)=H(t) *\left(K_{3} E_{o}(t)+K_{4} E_{o}^{*}(t)\right) .
$$

Substituting $E_{r x}(t)$ in Eqn. 9, then the received current is given as

$$
\begin{aligned}
I_{r x}(t) & =\left(K_{1} K_{3} E_{o}(t)+K_{1} K_{4} E_{o}^{*}(t)\right) * H(t) \\
& +\left(K_{2} K_{4}^{*} E_{o}(t)+K_{2} K_{3}^{*} E_{o}^{*}(t)\right) * H^{*}(t) .
\end{aligned}
$$

In the above equations, we have considered the photodetector responsivity to be unity. From Eqn. (18), we can deduce that the effect of IQ imbalance is neither commutative nor associative in the presence of channel and other transceiver phase impairments. Therefore, to compensate for the transceiver imbalance, we sequence the signal processing algorithm to compensate the receiver IQ imbalance up front, followed by equalization of the time-varying channel and transceiver impairments and finally correcting for transmitter IQ imbalance. Since the effect of transceiver IQ imbalance is time-dependent, the use of a joint multi-impairment signal processing for the equalization would be propitious.

\section{THEORY OF WIDELy LiNEAR FILTER}

Widely Linear (WL) Filter augments signal and its conjugate for extracting and utilizing the complete second-order statistics of the signal. Using the same principle, widely linearconstant modulus algorithm (WL-CMA) based complex filter augments signal and its conjugate for both $\mathrm{X}$ and $\mathrm{Y}$ polarization, respectively, for joint compensation of IQ Imbalance (or any non-circularity) and polarization mixing.

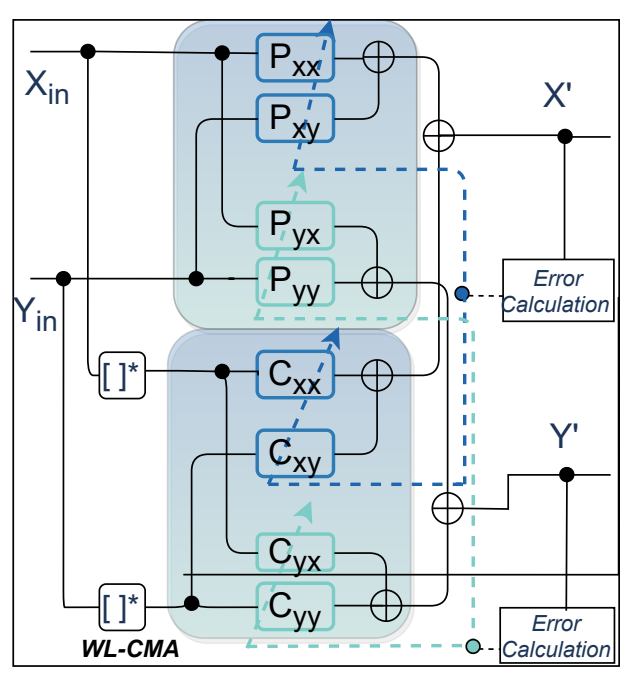

Fig. 3: Widely Linear Complex Filter

Figure 3 represents the block diagram of the WL-CMA filter where all the filter taps are updated by standard CMA/radius directed-CMA algorithm for M-QAM coherent optical systems [20]. The WL-CMA complex filter can effectively compensate for IQ imbalance and polarization mixing in the absence of laser phase noise in an optically dispersion compensated system. Detailed analysis of the WL-CMA filter is discussed 


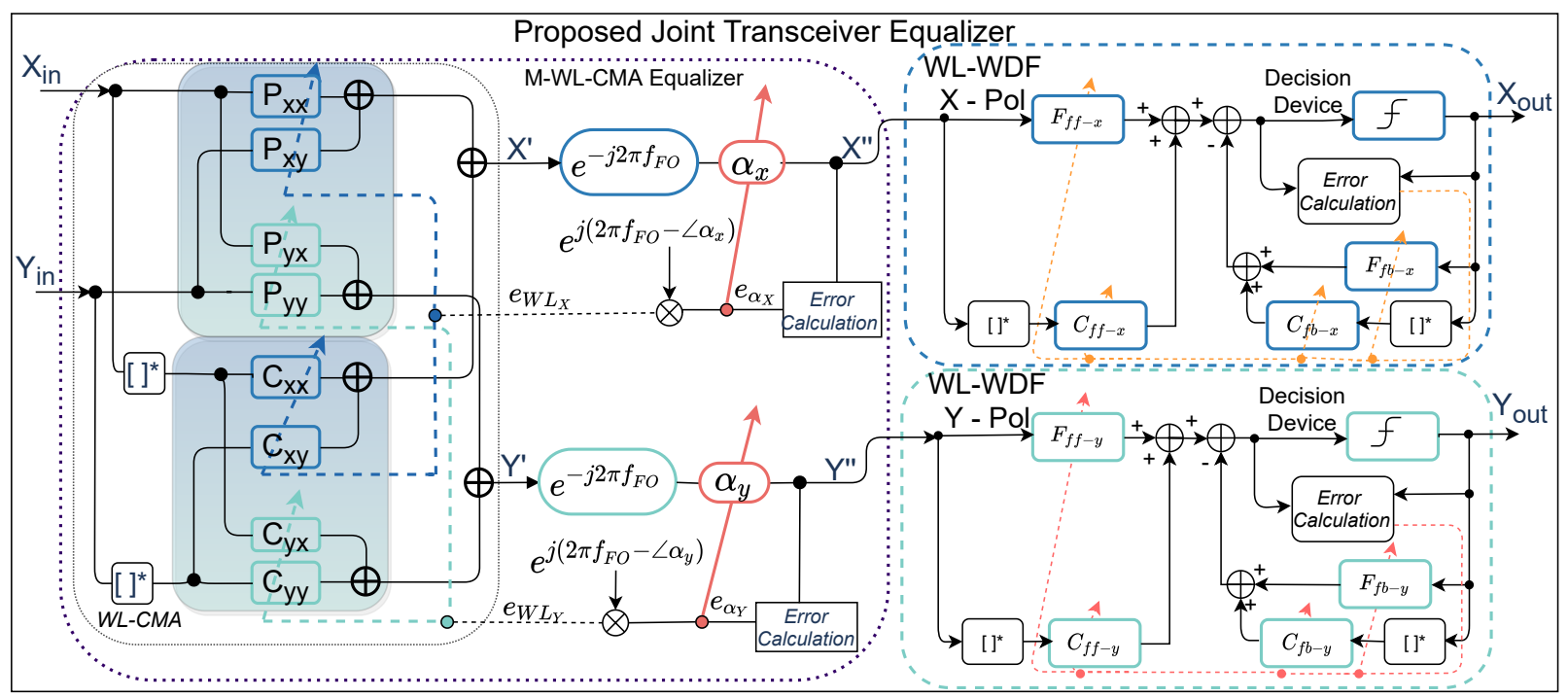

Fig. 4: Architecture of the proposed joint adaptive equalizer for multi-impairment compensation

for receiver imbalance in the absence of phase noise [20]. The WL-CMA complex filter with $2 N_{c d}$ taps can effectively compensate the receiver IQ imbalance in the presence of dispersion, where, $N_{c d}$ is the number of taps required for the dispersion compensation in time domain [28]. However, the conventional CMA or WL-CMA algorithms can only compensate for slow varying impairments such as skew, polarization mode dispersion, inter symbol interference due to bandwidth limitations and cannot compensate for the relatively fast varying impairments like frequency offset and phase noise, as no explicit phase information is provided to the equalizer. As discussed in section II-A, compensation of the receiver imbalance needs to be done before compensating any other impairments in optical communication systems. Also, $K_{1}$ and $K_{2}$ are fast time-varying parameters due to the presence of frequency offset, and phase noise and thereby, equalization with WL-CMA of fast time-varying phase impairments results in degraded performance; hence a joint equalization is required for compensation of receiver imbalance in the presence of laser phase noise as discussed and demonstrated in this paper. Similarly, $K_{3}$ and $K_{4}$ are fast time-varying IQ imbalance parameters and neither transmitter and receiver phase noise can be separated. Therefore, joint equalization is also a requisite for transmitter imbalance, and recently, we have demonstrated the joint compensation scheme for transmitter IQ imbalance in the presence of all other impairments in the optical communication system [23].

\section{JoInt TRANSCEIVER EQUaLIZER (JTE)}

Figure 4 shows the block diagram of our proposed algorithm for joint equalization of polarization mixing, receiver imbalance, and transceiver phase noise followed by compensation of transmitter imbalance. We refer to the proposed DSP sequence for dispersion-managed system as joint transceiver equalizer (JTE). As discussed in section II, commutative property of transmitter and receiver IQ imbalance breaks due to the different origins of IQ imbalance and impairments in optical communication systems such as dispersion, frequency offset and laser phase noise. Therefore, the transmitter and receiver IQ imbalance needs to be compensated separately. We have also discussed that transmitter and receiver IQ imbalance parameters are time-dependent, and hence, a joint equalization is required. Therefore, we first jointly compensate receiver IQ imbalance along with carrier recovery of the signal, followed by transmitter imbalance compensation.

A coarse value for frequency offset is estimated apriori using the conventional $4^{\text {th }}$ power periodogram technique and the estimated offset is provided as an implicit parameter to the adaptive equalizer for coarse frequency offset correction. Received samples are first normalized to unit average power and are then passed through the single-tap WL-CMA filter for joint compensation of receiver IQ Imbalance and polarization mixing, followed by the frequency offset compensation using the estimated frequency offset value. The samples are further passed through $\alpha_{x}$ and $\alpha_{y}$ filters for carrier phase tracking of $\mathrm{X}$ and $\mathrm{Y}$ polarization, respectively. The error estimation for the $\alpha_{x}$ and $\alpha_{y}$ filters are based on modified CMA criterion, where the values are estimated independently from the real and imaginary parts of the signal. Error signal for WL-complex filter is calculated by multiplying the phase information to the error signal for $\alpha_{x}$ and $\alpha_{y}$ filter. All the filter taps are updated independently using the standard CMA algorithm.

Update equations of the proposed adaptive equalizer are as follows,

$$
\begin{aligned}
& P_{x x}(n+1)=P_{x x}(n)+\mu_{1} e_{W L-X}(n) X_{i n}^{*}(n) \\
& P_{x y}(n+1)=P_{x y}(n)+\mu_{1} e_{W L-X}(n) Y_{i n}^{*}(n) \\
& P_{y x}(n+1)=P_{y x}(n)+\mu_{1} e_{W L-Y}(n) X_{i n}^{*}(n) \\
& P_{y y}(n+1)=P_{y y}(n)+\mu_{1} e_{W L-Y}(n) Y_{i n}^{*}(n) \\
& C_{x x}(n+1)=C_{x x}(n)+\mu_{1} e_{W L-X}(n) X_{i n}(n) \\
& C_{x y}(n+1)=C_{x x}(n)+\mu_{1} e_{W L-X}(n) Y_{i n}(n) \\
& C_{y x}(n+1)=C_{y x}(n)+\mu_{1} e_{W L-Y}(n) X_{i n}(n) \\
& C_{y y}(n+1)=C_{y y}(n)+\mu_{1} e_{W L-Y}(n) Y_{i n}(n)
\end{aligned}
$$




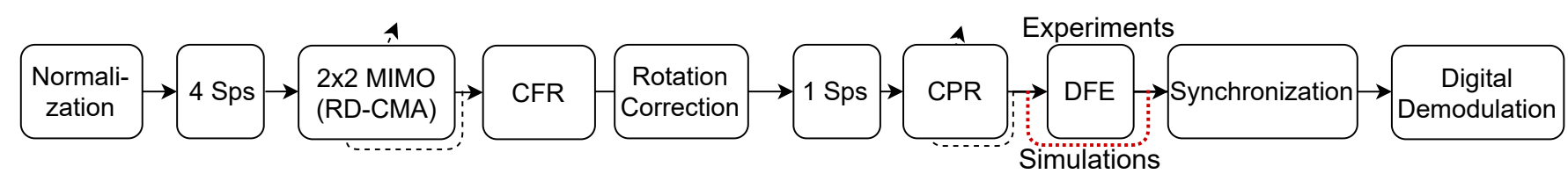

(a) Conventional DSP (C-DSP) (No IQ Imbalance Compensation)

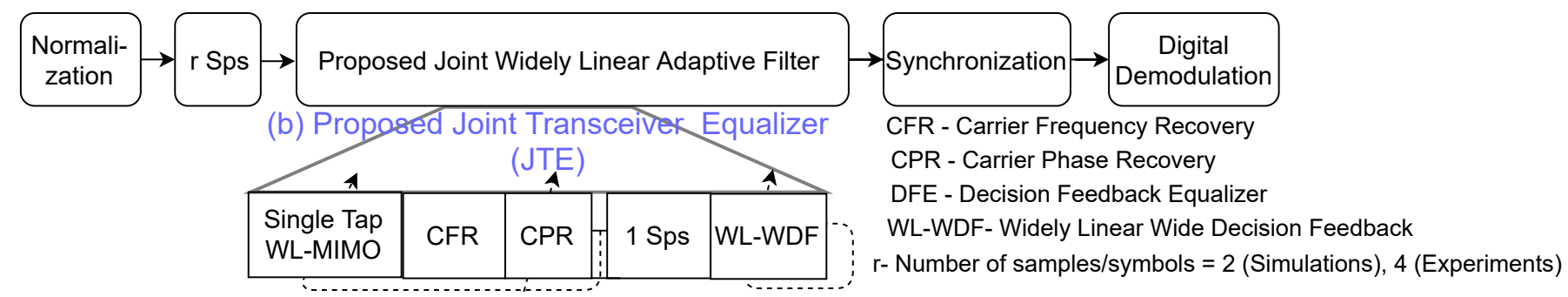

Fig. 5: (a) Conventional DSP sequence and (b) Proposed JTE Equalizer DSP sequence

where, $X_{i n}(n)$ and $Y_{i n}(n)$ are the input samples of $\mathrm{X}$ and $\mathrm{Y}$ polarization signals to the adaptive equalizer. Update equations of alpha filters are given as

$$
\begin{aligned}
& \alpha_{x}(n+1)=\alpha_{x}(n)+\mu_{2} e_{\alpha_{x}}(n) e^{-j 2 \pi n F_{f o}} X^{\prime}(n) \\
& \alpha_{y}(n+1)=\alpha_{y}(n)+\mu_{2} e_{\alpha_{y}}(n) e^{-j 2 \pi n F_{f o}} Y^{\prime}(n)
\end{aligned}
$$

where, $\mu_{1}$ and $\mu_{2}$ denote the step sizes of the WL-CMA and alpha filters, respectively. Typical values used for $\mu_{1}$ is $5 \times$ $10^{-4}$ and that of $\mu_{2}$ is $7 \times 10^{-3}$. However, values of step size can vary depending upon polarization mixing ratio, the variance of phase noise and IQ imbalance ratio. $X^{\prime \prime}$ and $Y^{\prime \prime}$ represent the carrier frequency and phase recovered $\mathrm{X}$-pol and Y-pol output signals of Modified WL-CMA (M-WL-CMA) filter where, the error signals $e_{\alpha_{x}}(n), e_{\alpha_{y}}(n), e_{W L-X}(n)$ and $e_{W L-Y}(n)$ are given as,

$$
\begin{aligned}
e_{\alpha_{x_{r}}}(n) & =X_{r}(n)\left\{\left|R_{x}(n)\right|^{2}-X_{r}^{2}(n)\right\} \\
e_{\alpha_{x_{i}}}(n) & =X_{i}(n)\left\{\left|R_{x}(n)\right|^{2}-X_{i}^{2}(n)\right\} \\
e_{\alpha_{x}}(n) & =e_{\alpha_{x_{r}}(n)}+j e_{\alpha_{x_{i}}(n)} . \\
e_{\alpha_{y_{r}}}(n) & =Y_{r}(n)\left\{\left|R_{y}(n)\right|^{2}-Y_{r}^{2}(n)\right\} \\
e_{\alpha_{y_{i}}}(n) & =Y_{i}(n)\left\{\left|R_{y}(n)\right|^{2}-Y_{i}^{2}(n)\right\} \\
e_{\alpha_{y}}(n) & =e_{\alpha_{y_{r}}}(n)+j e_{\alpha_{y_{i}}}(n) .
\end{aligned}
$$

$R_{x}(n)$ and $R_{y}(n)$ are the hard-decision radius for $\mathrm{X}$ and $\mathrm{Y}$ polarizations decided from the normalized radius of ideal M-QAM constellation. The terms $X_{r}(n), Y_{r}(n), X_{i}(n), Y_{i}(n)$ represent the real and imaginary components of $X^{\prime \prime}$ and $Y^{\prime \prime}$ signals respectively. Similarly, modified error signals provided to WL-CMA filter are given as

$$
\begin{aligned}
& e_{W L-X}(n)=e_{\alpha_{x}}(n) \exp \left(j\left(2 \pi n F_{f_{o}}-\angle \alpha_{x}(n)\right)\right) \\
& e_{W L-Y}(n)=e_{\alpha_{y}}(n) \exp \left(j\left(2 \pi n F_{f_{o}}-\angle \alpha_{y}(n)\right)\right)
\end{aligned}
$$

where, $\left(2 \pi n F_{f o}-\angle \alpha_{x / y}\right)$ represents the phase to be corrected by carrier recovery filters for the $X$ and $Y$ polarization signals, respectively. Therefore, providing the carrier phase information to the WL-CMA equalizer would help in equalizing polarization mixing and time-varying IQ imbalance parameters, since all the filters are updated concurrently.
Equalized signals, $X^{\prime \prime}$ and $Y^{\prime \prime}$ are passed independently through multitap widely linear wide decision feedback equalizer (WL-WDF) to compensate for transmitter imbalance and any residual ISI compensation for effective equalization of the signal. In a widely linear wide decision feedback filter, signal and its conjugate are passed through two independent feedforward multitap FIR filters and the sum of their outputs is taken as the feedforward signal. Similarly, decision signal and its conjugate is passed through two independent feedback multitap FIR filters, and the sum of their outputs are taken as the feedback signal. Output signal is realized by taking the sum of feedforward signal and feedback signal [29]. All the filters are updated based on standard minimum mean square error criterion, where the error signal is calculated from the hard decision ideal constellation points of the M-QAM signal.

Conventional DSP Sequence (C-DSP) is shown in Fig. 5 where the received signal is normalized and then resampled to 4 samples/symbol. The resampled signal is first passed through $2 \times 2$ MIMO RD-CMA equalizer followed by carrier frequency and phase recovery in a sequential manner. Since CMA is a blind and magnitude-based algorithm, signal after CMA equalizer suffers from phase rotation. Hence, to compensate the phase rotation, a phase de-rotator is employed in conventional DSP sequence. There are no bandwidth limitations considered in numerical simulation, and hence no DFE filter is used in simulation DSP sequence, whereas in experiments, components are bandwidth-limited and therefore a multi-tap DFE filter is employed for residual ISI compensation. Similarly, in JTE, the total number of feedforward and feedback taps in WL-WDF for simulations are considered as 3 for transmitter imbalance compensation, whereas for experiments the number of taps considered is 51 for the compensation of both transmitter imbalance and bandwidth limitations. In conventional DSP sequence, no explicit techniques are employed to compensate transmitter or receiver imbalance.

\section{Numerical Simulations}

We have numerically investigated the performance of the proposed joint transceiver equalizer and compared it with the 
conventional DSP sequence for polarisation multiplexed (PM) 16QAM and 64QAM systems. A schematic of the simulation setup for the transmission of 32 GBd PM-M-QAM is shown in Fig. 6. A PRBS-23 sequence is mapped to Mary-QAM symbols and a total of $2^{18}$ symbols (symbol rate: $32 \mathrm{GBd}$ and upsampled to $4 \mathrm{Sps}$ ), is used to drive a dual-polarization IQ-modulator to modulate on a laser $(1550 \mathrm{~nm}$, linewidth $12.5 \mathrm{kHz}$ ). Non-idealities such as phase imbalance and gain imbalance are introduced in the IQ modulator (Transmitter IQ Imbalance), as described in Eqn. (1) to study the effect of the same on the signal after transmission.

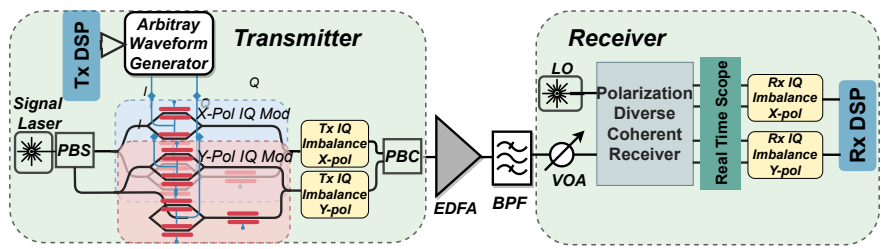

Fig. 6: Schematic of the simulation setup for M-QAM Coherent transmission system

Polarization transformation is modelled using the Jones matrix [30] and power loss in the system is compensated by an Erbium doped fiber amplifier (EDFA). Variation in optical signal to noise ratio (OSNR) is realized by changing the noise statistics in the EDFA. The optical signal is then coherently detected using an independent local oscillator laser (LO) of linewidth $12.5 \mathrm{kHz}$ with its frequency detuned with respect to the signal carrier frequency by $500 \mathrm{MHz}$. The phase noise of the transmitter laser and LO are generated using standard Wiener (random walk) process. Non-idealities at the receiver are modelled after the coherent receiver in the baseband domain. Received samples are normalized, and frequency offset is estimated using $4^{\text {th }}$ power periodogram technique followed by adaptive equalization as discussed in section IV. Signals in each polarization are then demodulated, and the received bits are used to evaluate the performance of the data in terms of $Q^{2}$, which is calculated from the measured bit error rate (BER) according to $Q^{2}(d B)=20 \times \log _{10}\left\{\sqrt{2} \operatorname{erfc} c^{-1}(2 B E R)\right\}$ [20].

\section{A. Effect of transceiver imbalance}

To study the effect of JTE and C-DSP on the transceiver imbalance, polarization mixing is maintained constant with power splitting ratio between two polarizations $(\beta)$ as 0.7 and phase delay between two polarizations $(\delta)$ as $\pi / 6$. Receiver gain and phase imbalance are also maintained constant at $\epsilon_{r x}=-0.05$, and $\phi_{r x}=5^{\circ}$ for PM-16QAM transmission and transmitter imbalance is varied. Figure 7 shows the $Q^{2}$ performance of PM-16QAM transmission versus transmitter phase imbalance for different transmitter gain imbalance values, $\epsilon_{t x}=-0.05,0$ and 0.05 at an OSNR $22 \mathrm{~dB}$ with a total linewidth of $50 \mathrm{kHz}$. Variation in $Q^{2}$ with JTE is about 0.21 $\mathrm{dB}$ with a mean value of $11.42 \mathrm{~dB}$ for the shown imbalance values whereas for C-DSP its about $1.16 \mathrm{~dB}$ around a mean $Q^{2}$ of $9.6 \mathrm{~dB}$. The JTE outperforms the conventional DSP over the range of IQ imbalances considered. The shaded part represents the region above HD-FEC limit, and hence a error free transmission is expected with additional FEC overhead.

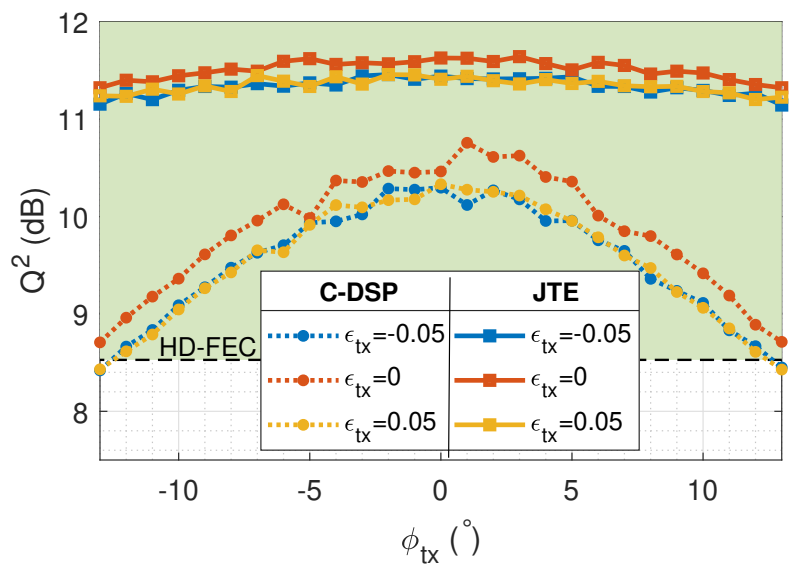

Fig. 7: $Q^{2}$ versus transmitter IQ imbalance for $32 \mathrm{GBd}$ PM 16QAM with $\epsilon_{r x}=-0.05$ and $\phi_{r x}=5^{\circ}$.

We further consider the PM-64QAM system with OSNR $28 \mathrm{~dB}$ and total linewidth of laser sources as $25 \mathrm{kHz}$, other parameters are the same as described above. We observe a similar performance of algorithms with receiver IQ imbalance values as $\epsilon_{r x}=0.025, \phi_{r x}=5^{\circ}$. Transmitter phase imbalance is varied from $-11^{\circ}$ to $11^{\circ}$ for different gain imbalance values as $\epsilon_{t x}=-0.025,0$ and 0.025 . Variation in $Q^{2}$ observed with JTE is about $0.17 \mathrm{~dB}$ with a mean value of $11 \mathrm{~dB}$ for the shown imbalance values, whereas for C-DSP it is about 1.98 $\mathrm{dB}$ around a mean $Q^{2}$ of $7.4 \mathrm{~dB}$.

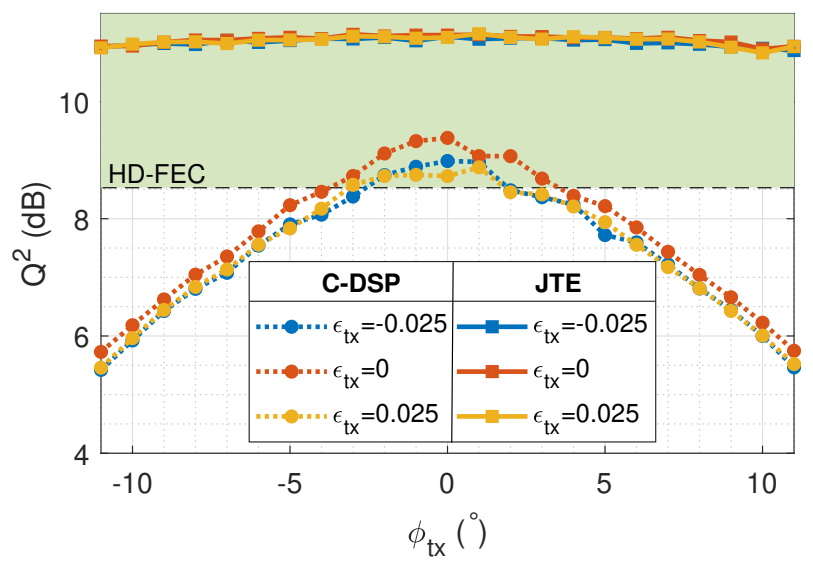

Fig. 8: $Q^{2}$ versus transmitter IQ imbalance for $32 \mathrm{GBd}$ PM 64QAM with $\epsilon_{r x}=-0.025$ and $\phi_{r x}=5^{\circ}$.

Figures 7 and 8 indicate that the improvement in $Q^{2}$ values is large for larger imbalance values in higher modulation systems. JTE also exhibits flatness for large variations in imbalance values and in both modulation formats. Numerical simulations are carried out for different receiver gain and phase imbalances, and our proposed DSP exhibits similar flatness in performance. 


\section{B. Effect of polarization mixing}

We have further investigated the proposed algorithm's performance for different polarization mixing conditions in presence of all other transceiver impairments. Figure 9 shows the $Q^{2}$ performance for different values of $\beta$, (denotes the power-splitting ratio) and $\delta$, (the phase difference) between $\mathrm{X}$ and $\mathrm{Y}$ polarizations for a $32 \mathrm{GBd}$ PM-64QAM systems with $\epsilon_{t x}=\epsilon_{r x}=0.025$ and $\phi_{t x}=\phi_{r x}=5^{\circ}$ at an OSNR of $28 \mathrm{~dB}$. We observe that the performance of C-

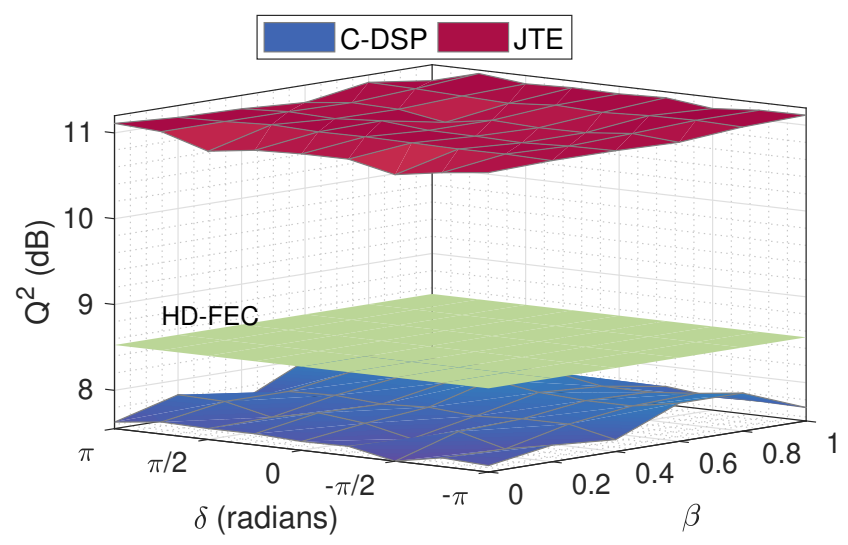

Fig. 9: $Q^{2}$ performance versus $\beta$ and $\delta$ for 32 GBd PM64QAM with $\epsilon_{t x}=\epsilon_{r x}=0.025$ and $\phi_{t x}=\phi_{r x}=5^{\circ}$.

DSP is below HD-FEC limits for any polarization mixing conditions, whereas JTE performance is approximately flat for all polarization mixing conditions, with a $Q^{2}$ improvement of $\approx 3 \mathrm{~dB}$ over C-DSP for all polarization mixing conditions. We have further investigated the algorithms for different IQ imbalance conditions in both the polarization as they may be different in real-world applications.

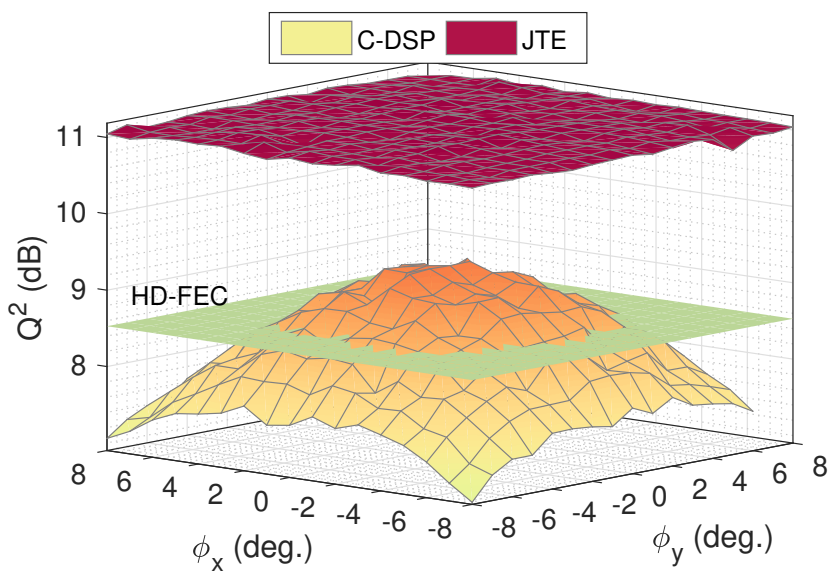

Fig. 10: $Q^{2}$ for different transmitter phase imbalance in $\mathrm{X}$ and Y polarizations for $32 \mathrm{GBd}$ PM-64QAM system.

Figure 10 shows the $Q^{2}$ performance with constant IQ imbalance parameters as $\phi_{r x}=5^{\circ}, \epsilon_{r x}=\epsilon_{t x}=0$ and transmitter phase imbalance is varied in both the polarizations for PM-64QAM systems at an OSNR of $28 \mathrm{~dB}$ with a total linewidth of $25 \mathrm{kHz}$. Polarization mixing parameters are considered as constant with $\beta=0.7$ and $\delta=\pi / 6$. We observe that our proposed algorithm can compensate the transceiver IQ imbalance even when the phase imbalance in both the polarizations are very different, whereas conventional DSP shows a poorer performance compared to JTE and varies with the phase imbalance introduced in both the polarization.

\section{Effect of linewidth of lasers}

We now investigate the influence of laser linewidth on the performance of the JTE and conventional DSP algorithms in the presence of transceiver and dispersion-managed channel impairments. Fig. 11 shows the performance of JTE compared with C-DSP for different linewidths with a constant parameters as $\epsilon_{t x}=0.05, \epsilon_{r x}=0.05, \phi_{t x}=5^{\circ}$ and $\phi_{r x}=5^{\circ}$ at an OSNR of $22 \mathrm{~dB}$ for PM-16QAM signals and $\epsilon_{t x}=0.025$, $\epsilon_{r x}=0.025, \phi_{t x}=5^{\circ}$ and $\phi_{r x}=5^{\circ}$ at an OSNR of $28 \mathrm{~dB}$ for PM-64QAM signals, respectively.

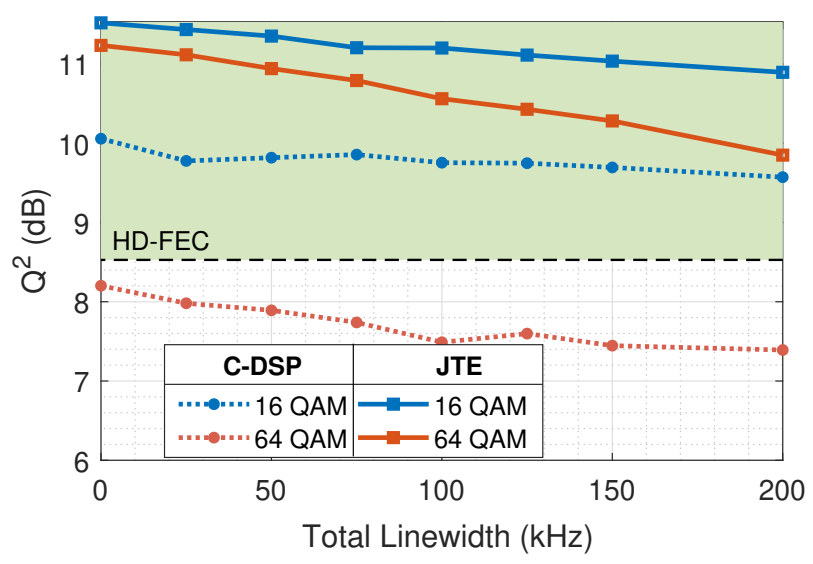

Fig. 11: $Q^{2}$ performance as a function of linewidth. Here, considered values are as $\phi_{t x} \& \phi_{r x}=5^{\circ}$, and $\epsilon_{t x} \& \epsilon_{r x}=$ 0.05 for PM-16QAM at an OSNR of $22 \mathrm{~dB}$ and $\epsilon_{t x} \& \epsilon_{r x}=$ 0.025 for PM-64QAM at an OSNR of $28 \mathrm{~dB}$.

As discussed in section II, receiver phase noise needs to be compensated before compensation of transmitter imbalance and transmitter phase noise needs to be compensated after transmitter imbalance compensation. Since the effect of phase noise due to signal laser and local oscillator on the received signal is indistinguishable, the performance of JTE degrades with the increase in the linewidth values. The phase margin decreases as we go for higher order cardinalities of modulation, thus decrease in performance with an increase in linewidth is more for PM-64QAM systems compared to PM16QAM systems. Nevertheless, results from Fig. 11 indicates that the performance of the proposed JTE algorithm is superior to the C-DSP algorithm for a wide range of considered linewidth values and for both the modulation formats. We also observed that the total linewidth tolerance of the proposed JTE algorithm for both PM-16QAM and PM-64QAM signals are much more than $200 \mathrm{kHz}$ for their performance to be within the HD-FEC limits. 


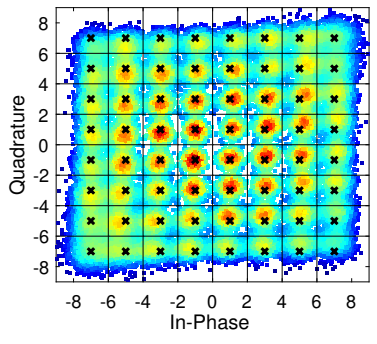

(a) $Q^{2}=6.07 \mathrm{~dB}$

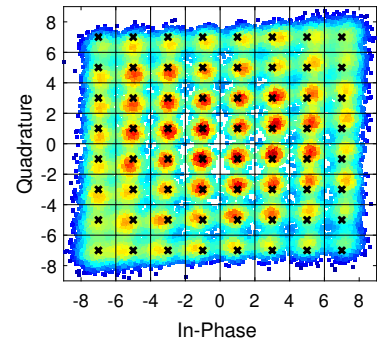

(b) $Q^{2}=6.01 \mathrm{~dB}$

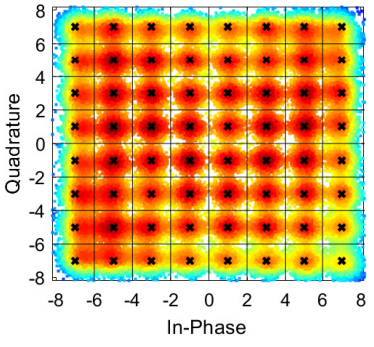

(c) $Q^{2}=9.69 \mathrm{~dB}$

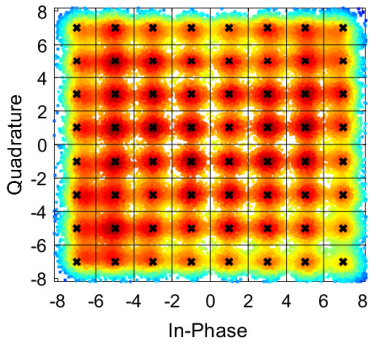

(d) $Q^{2}=9.50 \mathrm{~dB}$

Fig. 12: Constellation of the 16 GBd PM-64QAM signal processed using C-DSP algorithm (a) X-pol, (b) Y-pol, and proposed JTE algorithm (c) X-pol, (d) Y-pol with IQ imbalance parameters as $\epsilon_{r x}=0.1, \phi_{r x}=5^{\circ}, \epsilon_{t x}=0.025$ and $\phi_{t x}=6^{\circ}$.

\section{EXPERIMENTAL SETUP AND RESULTS}

We now validate the numerical results obtained in the previous sections through experimental demonstration. The schematic of the experimental setup is similar to that of the numerical simulation as shown in Fig. 6. The signal of desired modulation format (16QAM/64QAM) at appropriate symbol rate and with desired transmitter gain and phase imbalance is generated in the offline DSP. The generated signal is then loaded on an arbitrary waveform generator (AWG, 64GS/s, $23 \mathrm{GHz}$ ). The electrical channels at the output of AWG drive the dual-polarization IQ modulator, and the signal is modulated on a laser $(1550 \mathrm{~nm}, \approx 25 \mathrm{kHz}$ linewidth). The ASE noise is loaded into the polarization emulated M-QAM signal to get the required OSNR in $12.5 \mathrm{GHz}$ standard reference bandwidth and is connected to the phase and polarization diverse coherent receiver followed by real time scope $(80 \mathrm{GS} / \mathrm{s}$, $36 \mathrm{GHz}$ ). Receiver gain and phase imbalance is added to the digital samples offline, and then equalization is carried out as described in the previous section.

Figures 12 (a) and (b) show the final recovered constellations of the 16 GBd PM-64QAM signal processed with C-DSP algorithms and Fig. 12 (c) and (d) for signal processed with JTE of X and Y polarization respectively with IQ imbalance parameters as $\epsilon_{r x}=0.1, \phi_{r x}=5^{\circ}, \epsilon_{t x}=0.025$ and $\phi_{t x}=6^{\circ}$ at an OSNR of $32 \mathrm{~dB}$. The average improvement in $Q^{2}$ with the JTE compared to C-DSP is approximately $3.56 \mathrm{~dB}$. This improvement can also be visually observed from the rhombic skewness in constellations plots processed with CDSP whereas the recovered constellations from JTE are fully equalized.

We further investigate the performance for different values of transceiver imbalance. Figure 13 shows the $Q^{2}$ performance of PM-16QAM IQ imbalanced signals for $32 \mathrm{GBd}$ system at an OSNR of $24 \mathrm{~dB}$. Receiver gain imbalance $\epsilon_{r x}=-0.1$ and phase imbalance $5^{\circ}$ is maintained constant and transmitter imbalance is varied. Results in Fig. 13 experimentally demonstrate that the proposed JTE algorithm outperforms the C-DSP algorithm for all the considered imbalance values. We observed a significant improvement in $Q^{2}$ of $\approx 1.5 \mathrm{~dB}$ with $\epsilon_{t x}=-0.05, \epsilon_{r x}=-0.1$ and $\phi_{t x}=9^{\circ}, \phi_{r x}=5^{\circ}$. The variation in $Q^{2}$ observed for the proposed JTE algorithm is $0.26 \mathrm{~dB}$ and for the C-DSP algorithm is $0.68 \mathrm{~dB}$, clearly

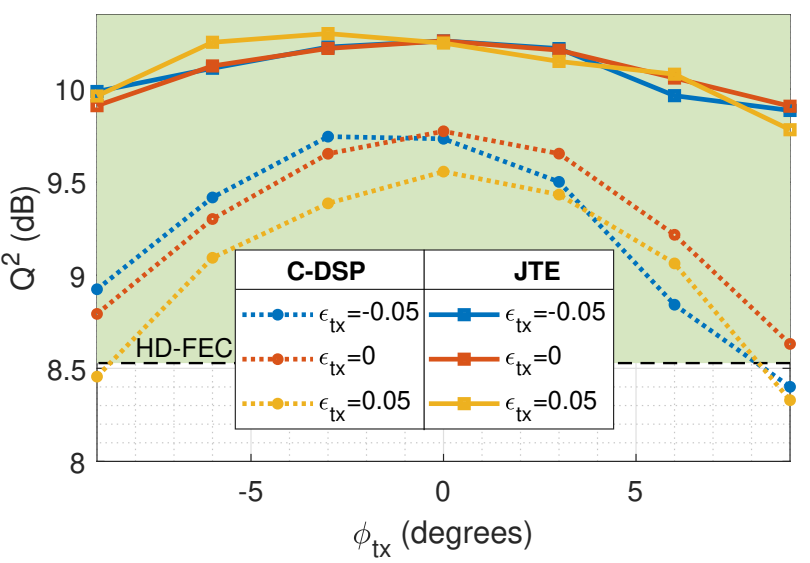

Fig. 13: $Q^{2}$ versus transmitter gain and phase imbalance for 32 GBd PM-16QAM with $\epsilon_{r x}=-0.1$ and $\phi_{r x}=5^{\circ}$.

indicating better flatness in the $Q^{2}$ performance of the JTE algorithm for various values of gain and phase imbalances.

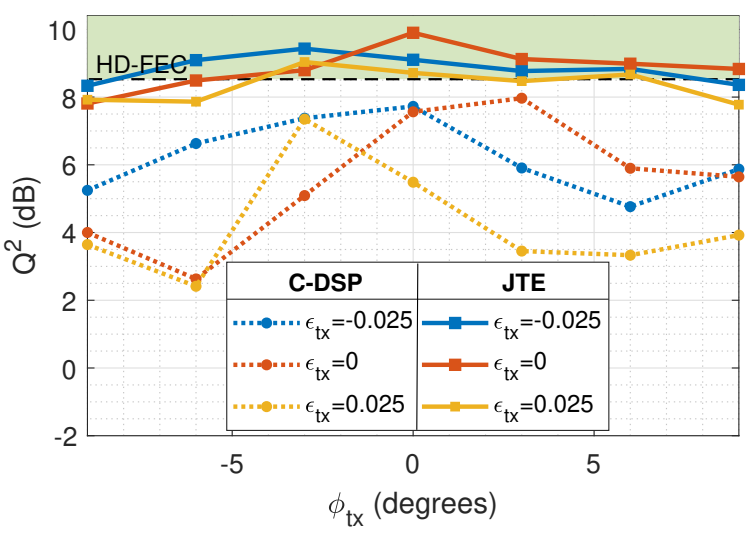

Fig. 14: $Q^{2}$ versus transmitter gain and phase imbalance for 16 GBd PM-64QAM with $\epsilon_{r x}=0.1$ and $\phi_{r x}=5^{\circ}$.

Figure 14 shows the $Q^{2}$ performance of PM-64QAM IQ imbalanced systems for $16 \mathrm{GBd}$ transmission at an OSNR of $32 \mathrm{~dB}$. Receiver gain imbalance $\left(\epsilon_{r x}\right)=0.1$ and phase imbalance $\left(\phi_{r x}\right)=5^{\circ}$ is maintained constant and transmitter 
IQ imbalance is varied. JTE outperforms C-DSP over a wide range of considered IQ imbalance values and performance of JTE is within HD-FEC limits for $\phi_{t x}=-4^{\circ}$ to $4^{\circ}$. We have observed a large improvement in $Q^{2}$ of $\approx 4 \mathrm{~dB}$ for $\epsilon_{t x}=$ $0, \epsilon_{r x}=0.1$ and $\phi_{t x}=9^{\circ}, \phi_{r x}=5^{\circ}$. Variation in $Q^{2}$ is $\approx 1.06 \mathrm{~dB}$ for JTE, whereas its about $2.69 \mathrm{~dB}$ for C-DSP, exhibiting flatness by JTE for considered imbalance values. This proves that our proposed transceiver DSP is tolerant to bandwidth limitations (leading to ISI) and thereby, tolerant to inter symbol interference. The performance of the proposed JTE algorithm is verified, and similar results are obtained for different values of receiver IQ imbalance.

\section{COMPARISON OF CONVERGENCE AND COMPLEXITY OF ALGORITHMS}

We compare the rate of convergence of proposed joint adaptive equalizers with conventional equalizers for $16 \mathrm{GBd}$ PM-64QAM experimental data transmission with transceiver imbalance parameters as $\epsilon_{t x}=0.025, \epsilon_{r x}=0.1, \phi_{t x}=6^{\circ}$ and $\phi_{r x}=5^{\circ}$ at an OSNR of $32 \mathrm{~dB}$. Fig. 15 shows the mean square error of the M-WL-CMA filter $\left(e_{W L-X / Y}\right)$ and CMA filter with a step size of $5 \mathrm{e}-4$ versus sample index for signals in $\mathrm{X}$ and $\mathrm{Y}$ polarizations respectively. The mean square error (MSE) is evaluated from the error calculated from hard decision samples and averaged over 1000 samples. The indicators $N_{c 1}$ and $N_{c 2}$ in Fig. 15 represent the number of samples required for convergence of CMA and M-WL-CMA filters for both polarizations respectively. The step size used for CMA and M-WL-CMA equalizer is 5e-4. We observe that the proposed joint adaptive M-WL-CMA equalizer exhibits faster convergence than CMA adaptive equalizer. Faster convergence is attributed to the joint equalization, and all the filter taps are updated concurrently; hence, the residual error is decaying faster and free of phase errors. However, the magnitude of MSE for M-WL-CMA is almost $3 \mathrm{~dB}$ larger than the CMA equalizer, and this is due to independent error calculations of real and imaginary parts of the signal. Nevertheless, increased residual MSE does not lead to degradation in the performance.

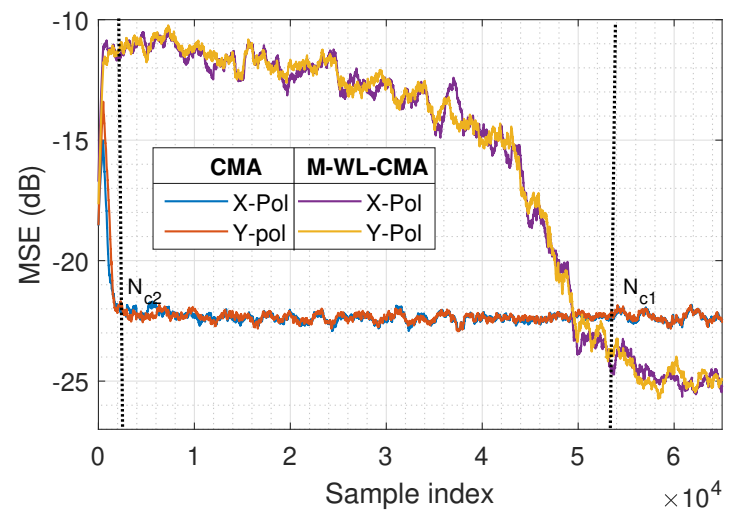

Fig. 15: MSE Convergence of MWLCMA and CMA Equalizers for PM-64QAM systems with $\epsilon_{t x}=0.025, \epsilon_{r x}=0.1$, $\phi_{t x}=6^{\circ}$ and $\phi_{r x}=5^{\circ}$.

\section{Complexity Analysis}

The details of complex operations that are different between C-DSP and JTE are discussed in Appendix.

\begin{tabular}{|c|c|c|}
\hline \# of Operations & C-DSP & JTE \\
\hline Additions & $10,53,200+10 N$ & $24,96,000+126 N$ \\
\hline Multiplications & $10,34,600+128 N$ & $24,84,000+252 N$ \\
\hline Conjugations & $9,26,600$ & $4,000+118 N$ \\
\hline
\end{tabular}

TABLE I: Complex Operations required in C-DSP and JTE, $N$ represents the number of processed samples

We found that for the 16 GBd PM-64QAM experimental data transmission with transceiver imbalance parameters as $\epsilon_{t x}=0.025, \epsilon_{r x}=0.1, \phi_{t x}=6^{\circ}$ and $\phi_{r x}=5^{\circ}$, the number of samples required for convergence are as $N_{c 1}=54000$, $N_{c 2}=2000, N_{c 3}=1300, N_{c 4}=12000, N_{c 5}=8000$, where $N_{c 1}, N_{c 2}, N_{c 3}, N_{c 4}, N_{c 5}$ are same as defined in Appendix. The number of taps in the multitap WL-WDF filter and DFE filter considered are 51 in both the polarizations. For the considered case, we have shown the convergence plot in Fig. 15 and final constellations in Fig. 12. The number of samples per symbol of each algorithm considered for calculating complexity is the same as mentioned in Fig. 5. However, number of samples required for convergence can vary depending upon different parameters like phase noise, mixing conditions, environmental fluctuations, etc. The total number of complex operations in C-DSP and JTE are shown in Table I for the considered case. We observe that the number of operations required by JTE is much higher than C-DSP, but it could be preferred due to its improved performance.

\section{CONCLUSIONS}

In this paper, we have shown that the impact of transmitter and receiver IQ imbalances are different in coherent optical communication system, and both needs to be compensated at different stages, especially in the presence of phase noise and frequency offset between the transmitter and receiver. The proposed blind JTE algorithm employing M-WL-CMA and WL-WDF equalizer effectively compensates the transceiver imbalances over a broader range of imbalances in the presence of other transceiver and channel impairments in an optically dispersion-managed link. We have investigated the proposed scheme through extensive numerical simulations for $32 \mathrm{GBd}$ PM-16QAM and PM-64QAM systems over a wider range of phase imbalances from -10 to 10 degrees and also verified the same through experiments for $32 \mathrm{GBd}$ PM-16QAM and 16 GBd PM-64QAM systems. Experimental results are corroborated with numerical simulations, a larger improvement is observed for higher order modulation cardinalities. The number of FIR filters are doubled in WL-CMA and WL-WDF as compared to CMA and DFE, thereby increasing the number of multiplications and additions in JTE as compared to C-DSP. Though the complexity of JTE is higher than C-DSP, it could still be preferred due to its improved performance and faster convergence for all the gain and phase imbalance values. Also, JTE can be carried out in a parallel implementation, hence latency remains the same as that of C-DSP. The proposed scheme has a wide application in diagnosing and correcting for transceiver imbalances and skew in networks. 


\begin{tabular}{|c|c|c|c|}
\hline \multicolumn{4}{|c|}{ M-WL-CMA Filter } \\
\hline Process & \# of Additions & \# of Multiplications & \# of Conjugation \\
\hline $\mathrm{X}^{\prime}$ and Y' Calculation & $6 N$ & $8 N$ & $4 N$ \\
\hline X" and Y" Calculation & 0 & $4 N$ & 0 \\
\hline$e_{\alpha_{x}}$ and $e_{\alpha_{y}}$ Calculation & $2 N_{c 2}$ & $2 N_{c 2}$ & 0 \\
\hline$e_{W L_{X}}$ and $e_{W L_{Y}}$ Calculation & 0 & $6 N_{c 2}$ & 0 \\
\hline WL-Filter tap updates & $8 N_{c 2}$ & $8 N_{c 2}$ & 0 \\
\hline$\alpha$ Fiter tap updates & $2 N_{c 2}$ & $2 N_{c 2}$ & $2 N_{c 2}$ \\
\hline Total Operations (a) & $12 N_{c 2}+6 N$ & $18 N_{c 2}+12 N$ & $2 N_{c 2}+4 N$ \\
\hline \multicolumn{4}{|c|}{ WL-WDF Filter for both polarizations } \\
\hline$X_{\text {out }}$ and $Y_{\text {out }}$ Calculation & $2 N_{\text {taps }} * N$ & $2 N_{\text {taps }} * 2 N$ & $2 N_{\text {taps }} * N$ \\
\hline Error signal Calculation & $2 N_{c 4}$ & 0 & 0 \\
\hline$F_{f f}$ and $F_{f b}$ Filter tap updates & $2 N_{\text {taps }} * N_{c 4}$ & $2 N_{t a p s} * N_{c 4}$ & 0 \\
\hline$C_{f f}$ and $C_{f b}$ Filter tap updates & $2 N_{\text {taps }} * N_{c 4}$ & $2 N_{\text {taps }} * N_{c 4}$ & 0 \\
\hline Total Operations (b) & $2 N_{\text {taps }} *\left(N+2 N_{c 4}\right)+2 N_{c 4}$ & $2 N_{t a p s} *\left(2 N+2 N_{c 4}\right)$ & $2 N_{\text {taps }} * N$ \\
\hline \multicolumn{4}{|c|}{ C-DSP for both polarizations } \\
\hline Total Operations in CMA & $4 N_{c 1}+2 N$ & $4 N_{c 1}+4 N$ & $2 N_{c 1}$ \\
\hline Operations in carrier frequency compensation & 0 & $2 \mathrm{~N}$ & 0 \\
\hline Operations in phase rotation correction & $2 \mathrm{~N}$ & $2 \mathrm{~N}$ & 0 \\
\hline Total Operations in DD-LMS (X and Y pol) & $4 N_{c 3}$ & $2 N_{c 3}+2 N$ & $2 N_{c 3}$ \\
\hline Total Operations in DFE (X and Y pol) & $\left(2 N_{t a p s}+2\right) * N_{c 5}$ & $\left(2 N_{c 5}+2 N\right) * N_{\text {taps }}$ & $2 N_{t a p s} * N_{c 5}$ \\
\hline
\end{tabular}

TABLE II: Complexity Analysis of proposed JTE and C-DSP

\section{ACKNOWLEDGMENT}

Funding from Office of the Principal Scientific Advisor, Ministry of Electronics and Information Technology and Ministry of Human Resource Development, Government of India is acknowledged. The authors would also like to acknowledge insightful discussions with Dr Edson Porto Da Silva.

\section{APPENDIX}

We have calculated the number of complex operations for the algorithms that are different in conventional DSP and JTE. Table II shows the number of complex operations in the MWL-CMA filter and WL-WDF filter for X-Pol. $N_{c 2}$ and $N_{c 4}$ denotes the number of symbols required for convergence in $\mathrm{M}$ WL-CMA filter and WL-WDF filter. $N_{\text {taps }}$ denotes the total number of taps in $F_{f f}$ and $F_{f b}$ of the multitap WL-WDF filter. An equal number of taps are employed in the conjugate signal of WL-WDF filter, $N_{\text {taps }}=C_{f f}+C_{f b}$. Similarly, we have calculated the total number of operations in C-DSP. $N_{c 1}, N_{c 3}$ and $N_{c 5}$ denote the number of samples required for convergence of CMA, DD-LMS and DFE filter, respectively, in C-DSP. The $\mathrm{N}$ represents the number of samples processed in a given block.

\section{REFERENCES}

[1] Galdino, Lidia and Edwards, Adrian and Yi, Wenting and Sillekens, Eric and Wakayama, Yuta and Gerard, Thomas and Pelouch, Wayne Sheldon and Barnes, Stuart and Tsuritani, Takehiro and Killey, Robert I. and Lavery, Domaniç and Bayvel, Polina, "Optical Fibre Capacity Optimisation via Continuous Bandwidth Amplification and Geometric Shaping," in IEEE Photonics Technology Letters, vol. 32, no. 17, pp. 1021-1024, 2020.

[2] Chen, Xi and Cho, Junho and Che, Di, "Quantification of Implementation Penalties for Ultra-High Order QAM Systems," 2020 Opto-Electronics and Communications Conference (OECC), Taipei, Taiwan, 2020, pp. 1-2.

[3] X. Chen, J. Cho, A. Adamiecki and P. Winzer, "16384-QAM transmission at $10 \mathrm{GBd}$ over $25-\mathrm{km}$ SSMF using polarizationmultiplexed probabilistic constellation shaping," European Conference on Optical Communication (ECOC 2019), Dublin, Ireland, 2019, pp. 1-4.
[4] Xiaoxiao Dai, Xiang Li, Ming Luo, and Shaohua Yu, "Numerical simulation and experimental demonstration of accurate machine learning aided IQ time-skew and power-imbalance identification for coherent transmitters," Optics Express vol. 27, no. 26 (2019): 38367-38381.

[5] Yangyang Fan, Yufeng Jiang, Zhenning Tao, Hisao Nakashima, Takeshi Hoshida, "Calibration and monitoring of coherent optical transceiver imperfections" Proc. SPIE 11309, Next-Generation Optical Communication: Components, Sub-Systems, and Systems IX, 113090N 2020.

[6] Q. Zhang, Y. Yang, C. Gu, Y. Yao, A. P. T. Lau and C. Lu, "MultiDimensional, Wide-Range, and Modulation-Format-Transparent Transceiver Imbalance Monitoring," in Journal of Lightwave Technology, vol. 39, no. 7, pp. 2033-2045, 2021.

[7] C. M. Diniz, F. Da Ros, E. P. da Silva, R. T. Jones and D. Zibar, "Optimization of DP-M-QAM Transmitter Using Cooperative Coevolutionary Genetic Algorithm," in Journal of Lightwave Technology, vol. 36, no. 12, pp. 2450-2462, 2018.

[8] Trung-Hien Nguyen, Pascal Scalart, Mathilde Gay, Laurent Bramerie, Olivier Sentieys, Jean-Claude Simon, Christophe Peucheret, and Michel Joindot, "Blind Transmitter IQ Imbalance Compensation in M-QAM Optical Coherent Systems," Journal of Optical Communication Networks vol. 9, pp. D42-D50, 2017.

[9] T. Kobayashi, M. Nakamura, F. Hamaoka, M. Nagatani, H. Wakita, H. Yamazaki, T. Umeki, H. Nosaka, and Y. Miyamoto, "35-Tb/s C-band Transmission over $800 \mathrm{~km}$ Employing 1-Tb/s PS-64QAM signals enhanced by Complex $8 \times 2$ MIMO Equalizer," in Optical Fiber Communication Conference Postdeadline Papers 2019, paper Th4B.2.

[10] C. R. S. Fludger and T. Kupfer, "Transmitter Impairment Mitigation and Monitoring for High Baud-Rate, High Order Modulation Systems," European Conference on Optical Communication, Dusseldorf, Germany, 2016, pp. 1-3.

[11] M. Casabella, A. L. Pola and M. R. Hueda, "Reduced Complexity Compensation of I/Q Skew and Imbalance in Subcarrier Multiplexing Receivers," IEEE Photonics Conference (IPC), Vancouver, BC, Canada, 2020, pp. 1-2.

[12] Q. Xiang, Y. Yang, Q. Zhang, J. Cao and Y. Yao, "Compensation of IQ imbalance using Kalman filter in coherent optical systems," Opto-Electronics and Communications Conference (OECC), Jeju, Korea (South), 2018, pp. 1-2.

[13] Lagha, Marwa Kazdoghli and Gerzaguet, Robin and Bramerie, Laurent and Gay, Mathilde and Chares, Marie-Laure and Peucheret, Christophe and Scalart, Pascal, "Blind Joint Polarization Demultiplexing and IQ Imbalance Compensation for $M$ - 
QAM Coherent Optical Communications," in Journal of Lightwave Technology, vol. 38, no. 16, pp. 4213-4220, 2020.

[14] J.Liang,Y.Fan,Z.Tao,X.Su,and H.Nakashima, "Transceiver imbalances compensation and monitoring by receiver DSP," in Journal of Lightwave Technology,to be published (early access), doi: 10.1109/JLT.2019.2944830.

[15] M. Paskov, D. Lavery and S. J. Savory, "Blind Equalization of Receiver In-Phase/Quadrature Skew in the Presence of Nyquist Filtering," in IEEE Photonics Technology Letters, vol. 25, no. 24, pp. 2446-2449, 2013.

[16] P. Skvortcov, I. Phillips, and W. Forysiak, "Transmitter frequency-dependent IQ imbalance characterization and preemphasis," in OSA Advanced Photonics Congress 2020, paper SpTh1I.2.

[17] C. Li, Z. Xu, Q. Yang, and S. Yu, "Transmitter IQ Mismatch Compensation Based on Second Order Moment Estimation for Coherent Optical M-QAM System," in Asia Communications and Photonics Conference 2016, paper AF4B.1.

[18] M. S. Faruk and S. J. Savory, "Digital Signal Processing for Coherent Transceivers Employing Multilevel Formats," in Journal of Lightwave Technology, vol. 35, no. 5, pp. 1125-1141, 2017.

[19] Danilo P. Mandic, Vanessa Su Lee Goh,“ Complex Valued Nonlinear Adaptive Filters: Noncircularity, Widely Linear and Neural Models" New York, NY, USA: Wiley, 2009.

[20] Edson Porto da Silva and Darko Zibar, "Widely Linear Equalization for IQ Imbalance and Skew Compensation in Optical Coherent Receivers," Journal of Lightwave Technolgy 34, 35773586, 2016.

[21] E. P. d. Silva and D. Zibar, "Widely Linear Blind Adaptive Equalization for Transmitter IQ-Imbalance/Skew Compensation in Multicarrier Systems" European Conference on Optical Communication, Dusseldorf, Germany, 2016, pp. 1-3.

[22] Z. Sha and Z. Wang, "Channel Estimation and Equalization for Terahertz Receiver With RF Impairments," in IEEE Journal on Selected Areas in Communications, vol. 39, no. 6, pp. 1621-1635, June 2021.

[23] Rekha Yadav, Lakshmi Narayanan Venkatasubramani, R David Koilpillai, D. Venkitesh, "Modified Widely Linear Filter for Simultaneous Multi-impairment Compensation", in Conference on Lasers and Electro-Optics, OSA Technical Digest (Optical Society of America, 2021), paper-SF2D.3.

[24] Zhang, Qun and Yang, Yanfu and Guo, Changjian and Zhou, Xian and Yao, Yong and Lau, Alan Pak Tao and Lu, Chao, "Algorithms for Blind Separation and Estimation of Transmitter and Receiver IQ Imbalances,' in Journal of Lightwave Technology, vol. 37, no. 10, pp. 2201-2208, 2019.

[25] Yudong He, Xian Zhou, Jiahao Huo, Qun Zhang, Jinhui Yuan, "Joint estimation of transmitter and receiver IQ imbalances based on GSOP and GCM for optical coherent systems", Results in Optics, vol. 4, 2021.

[26] C. Ju, N. Liu and C. Li, "In-Service Blind Transceiver IQ Imbalance and Skew Monitoring in Long-Haul Non-Dispersion Managed Coherent Optical Systems," in IEEE Access, vol. 7, pp. 150051-150059, 2019.

[27] Manabu Arikawa and Kazunori Hayashi, "Transmitter and receiver impairment monitoring using adaptive multi-layer linear and widely linear filter coefficients controlled by stochastic gradient descent," Optic Express 29, 11548-11561, 2021.

[28] S. J. Savory, "Digital Coherent Optical Receivers: Algorithms and Subsystems," in IEEE Journal of Selected Topics in Quantum Electronics, vol. 16, no. 5, pp. 1164-1179, Sept.-Oct. 2010.

[29] D. Mattera, L. Paura and F. Sterle, "Widely linear decisionfeedback equalizer for time-dispersive linear MIMO channels," in IEEE Transactions on Signal Processing, vol. 53, no. 7, pp. 2525-2536, July 2005.

[30] Kazuro Kikuchi, "Performance analyses of polarization demultiplexing based on constant-modulus algorithm in digital coherent optical receivers," Optics Express 19, 9868-9880, 2011.
[31] Faruk, M.S. "Blind equalization and carrier-phase recovery based on modified constant-modulus algorithm in PDM-QPSK coherent optical receivers", Optics Quantum Electronics 48, 2016.

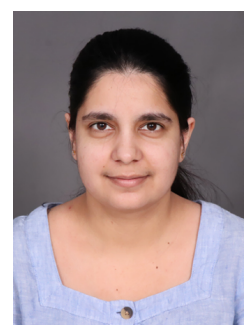

REKHA YADAV received the B.Tech. degree in Electronics Instrumentation and Control engineering from YMCA University of Science \& Technology, Faridabad, India, in 2017. She is currently pursuing the Master of Science by Research degree in Indian Institute of Technology Madras, Chennai, India. Her current research interests include digital signal processing, high-speed fiber optics communication.

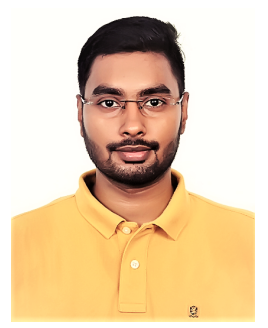

LAKSHMI NARAYANAN VENKATASUBRAMANI received the B.Tech. degree in Electronics and Communication Engineering from Amrita Vishwa Vidyapeetham University, Coimbatore, India, in 2014. He is currently pursuing the Ph.D.degree with Indian Institute of Technology Madras, Chennai, India. He was a short-term Visiting researcher with Dublin City University, Ireland, in Fall 2019. His current research interests include various topics in high-capacity optical communications and signal processing.

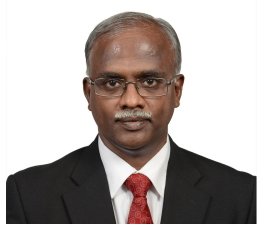

R. DAVID KOILPILLAI , is currently the Head of the Department and the Qualcomm Institute Chair Professor of electrical engineering, Indian Institute of Technology Madras, Chennai. Prior to joining IITM, he was with General Electric Corporate Research and Development for four years, and Ericsson, USA, for eight years, where he held different technical and managerial positions. In 2000, he became the Director of the Ericsson's Advanced Technologies and Research Department, RTP, NC, USA, where he developed GPRS/EDGE handset technology. His technical contributions at GE and Ericsson have resulted in 32 U.S. patents, ten Canadian Patents, and 19 WIPO/European patents. His current technical activities are in the areas of cellular evolution- $4 \mathrm{G}$ and $5 \mathrm{G}$, smart grid communications, and DSP for high-speed coherent optical communications.

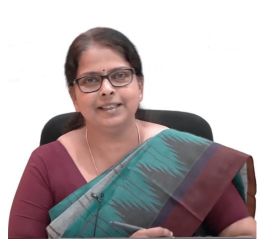

DEEPA VENKITESH, received her $\mathrm{PhD}$ in physics in 2009 from the Indian Institute of Technology Bombay, Mumbai, India. She is currently a professor at the Department of Electrical Engineering, Indian Institute of Technology Madras, Chennai, India. Her research interests include applications of nonlinear optics, optical signal processing for communication systems, and fiber lasers. She has authored more than 100 publications in international peer- reviewed journals and conferences. She is a Senior Member of the Optical Society and has been a frequent reviewer of several IEEE and OSA journals. She is currently an Associate Editor for the OSA journal, Advances in Optics and Photonics. 